TITLE:

\title{
Measuring the electron affinity of organic solids: an indispensable new tool for organic electronics.
}

$\operatorname{AUTHOR}(\mathrm{S})$ :

Yoshida, Hiroyuki

\section{CITATION:}

Yoshida, Hiroyuki. Measuring the electron affinity of organic solids: an indispensable new tool for organic electronics.. Analytical and bioanalytical chemistry 2014, 406(9-10): 22312237

ISSUE DATE:

2014-02-28

URL:

http://hdl.handle.net/2433/198619

\section{RIGHT:}

The final publication is available at Springer via http://dx.doi.org/10.1007/s00216-0147659-1.; This is not the published version. Please cite only the published version.; この論 文は出版社版でありません。引用の際には出版社版をご確認ご利用ください。 
Analytical and Bioanalytical Chemistry. 406, 2231-2237 (2014)

\title{
Measuring the electron affinity of organic solids: An indispensable new tool for organic electronics
}

\author{
Hiroyuki Yoshida
}

Institute for Chemical Research, Kyoto University, Uji, Kyoto 611-0011, Japan

yoshida@e.kuicr.kyoto-u.ac.jp

\begin{abstract}
Electron affinity is a fundamental energy parameter of materials. In organic semiconductors, the electron affinity is closely related with electron conduction. It is not only important to understand fundamental electronic processes in organic solids, but it is also indispensable for the research and development of organic semiconductor devices such as organic light emitting diodes (OLED) and organic photovoltaic cells (OPV). However, there has been no experimental technique for examining the electron affinity of organic materials that meets the requirement of such research. Recently a new method, called low-energy inverse photoemission spectroscopy, has been developed. A beam of low-energy electrons is focused onto the sample surface, emitting photons due to the radiative transition to unoccupied states which are then detected. From the onset of the spectral intensity, the electron affinity is determined within an uncertainty of $0.1 \mathrm{eV}$. Unlike the conventional

inverse-photoemission spectroscopy, sample damage is negligible and resolution is improved by a factor of two. The principle of the present method as well as several applications are discussed.
\end{abstract}

\section{Keywords}

Electron affinity, Ionization energy, Organic semiconductor, Low-energy inverse photoemission spectroscopy

\section{Introduction}

Ionization energy and electron affinity are fundamental energy parameters that characterize the property of materials. The ionization energy is defined as the minimum energy required to remove an electron out of a neutral atom or molecule in its ground state, while the electron affinity is the energy released when an additional electron is attached to a neutral atom or molecule. They are closely related to the reactivity and bonding nature of atoms and molecules [1]. The ionization energy and electron affinity of solid organic materials are also defined in a similar way [2].

Around 1950, some organic materials were discovered to have semiconducting properties [3-5]. Systematic studies of organic semiconductors started and, about four decades later, practical devices using organic semiconductors such as organic light emitting diodes (OLED) [6] and organic photovoltaic cells (OPV) [7] were reported. Now OLEDs are used as displays for mobile phones and portable digital media players, car radios and digital cameras. The power conversion efficiency of OPV has rapidly increased for the last few years and exceeded $10 \%$ in 2011 which was considered to be a milestone for the practical application of the technology [8].

In these devices, both holes and electrons which, respectively, carry positive and negative charges play a crucial role. When the one electron approximation is applied, the hole moves at the top of the valence levels while the electron moves at the bottom of the unoccupied states; the energies of these levels with respect to the vacuum level are the ionization energy and electron affinity, respectively. Figure 1 shows the energy level diagram of semiconductors and the experimental techniques used to examine their electronic structure. Electrons occupy the core levels and the valence states. The states which are not occupied by electrons are called unoccupied states. The difference in energy between the bottom of the unoccupied and the top of the occupied states is called the energy gap. The Fermi level lies somewhere in this energy gap[9].

The core and valence states have been extensively studies by photoemission spectroscopy (PES). In this technique, the sample is irradiated with a monochromatic photon $h v$ and the kinetic energies of the ejected electrons $E_{\mathrm{k}}$ are analyzed. From the energy conservation rule, the binding energy of the electron $E_{\mathrm{b}}$ is determined. The ionization energy is determined from the onset of the PES spectral intensity with respect to the vacuum level.

In contrast, the determination of unoccupied states and electron affinity are more difficult [10,11]. Often the energy gap is estimated from the onset of the optical transition between the valence and unoccupied states using ultraviolet-visible spectroscopy (photoabsorption spectroscopy; PAS). The electron affinity is calculated by adding the energy gap and the ionization energy. However, the energy gap determined by PAS is often smaller than the actual energy gap by 0.2 to $1 \mathrm{eV}$, and difference is interpreted as the exciton binding energy $[12,13]$. Thus the 

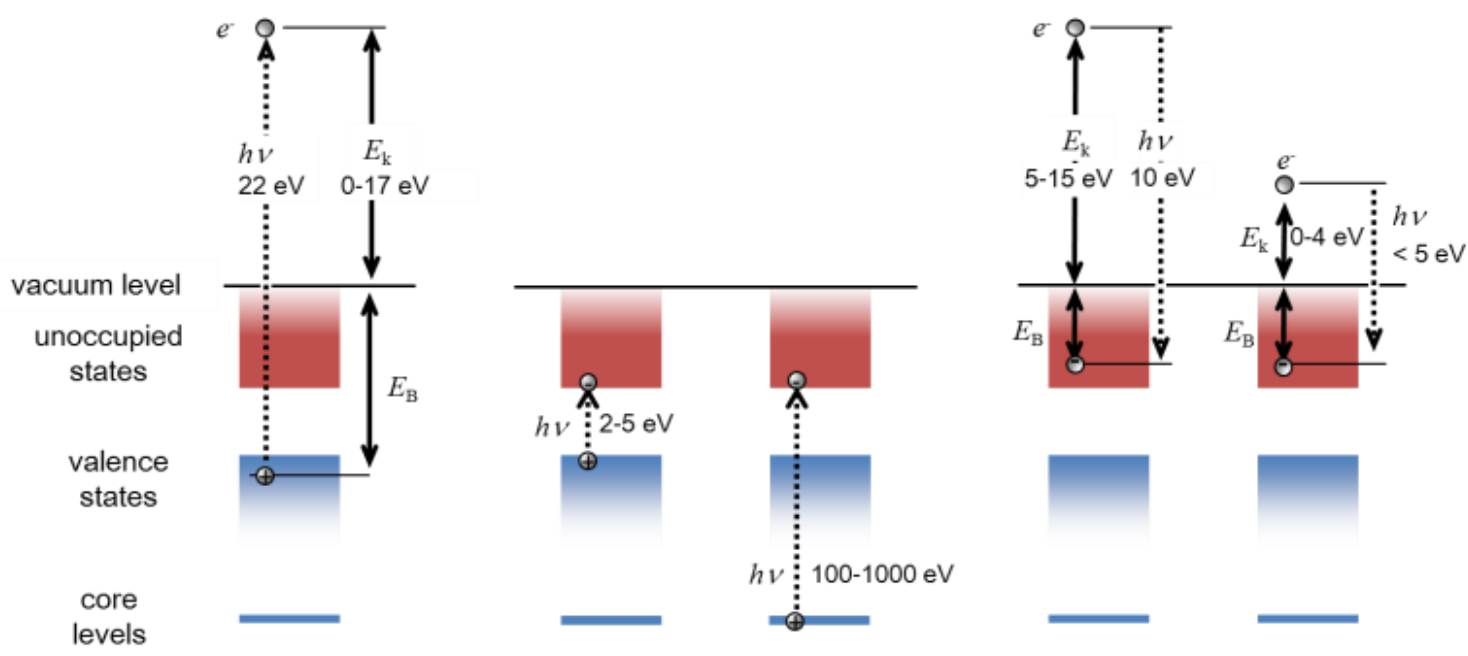

Fig 1:

Energy level diagram of experimental techniques for determining the ionization energy and electron affinity: photoemission spectroscopy (PES), ultraviolet-visible spectroscopy (photoabsorption spectroscopy; PAS), X-ray absorption spectroscopy (XAS), inverse photoemission spectroscopy (IPES), and low-energy inverse photoemission spectroscopy (LEIPS).

electron affinity is overestimated by PAS. X-ray absorption spectroscopy (XAS) uses the electronic transition from a core to an unoccupied state. The interaction between the excited electron and the core-hole generated by high energy X-ray largely affects the electronic states (core excitonic effect) preventing quantitative determination of the electron affinities.

In inverse photoemission spectroscopy (IPES), an electron having a kinetic energy $E_{\mathrm{k}}$ is incident to the sample and photons $h v$ emitted due to radiative transitions to the unoccupied states are detected. Inverse photoemission can be regarded as an inversion process of photoemission, from which the technique gets its name. The energy released by adding an electron to the sample is directly measured as the photon energy, which fits the definition of the electron affinity[14]. Since IPES is the most versatile and direct method to examine the unoccupied states and determine the electron affinities [10, 11, 15], the history and limitation of IPES is discussed in the next section.

There are other techniques, such as internal photoemission, electron transmission spectroscopy [11]. Although these are historically important, they can be applied only under limited conditions. For example, electron transmission can be applied to only material with a negative electron affinity. Scanning tunneling spectroscopy (STS) can also access the valence and unoccupied states of organic films [11, 16]; using scanning tunneling microscopy (STM) setup, the tip is fixed at the position of interest and the tunneling current is measured as a function of bias voltage to examine the density of states. The advantage of this technique is its extremely high spatial resolution down to sub-molecular size [17]. On the other hand, this method is applicable to only sub-monolayer films, while the damage to organic samples due to the high electron current density induced from the tip has not yet been clarified.

\section{Previous inverse photoemission spectroscopy (IPES)}

Although IPES is an ideal tool to examine the unoccupied states of solid materials in principle, it is far less frequently used than PES, mostly because the cross section of the IPES process is 3 or 5 orders of magnitude smaller than that of PES [15, 18]. In practical experiments, an intense electron beam is required and weak photon signals are detected.

IPES was first carried out in the X-ray range [10]. IPES became more widely used after the development of bandpass photon detectors for vacuum ultraviolet (VUV) light in the late 1970s by Dose [19, 20]. This type of photon detector has high sensitivity, high collection efficiency and simple construction. Figures $2 \mathrm{a}$ and $2 \mathrm{~b}$ show a typical IPES apparatus and the sensitivity curves for the bandpass photon detector, respectively. The detector consists of an iodine-filled Geiger Müller tube and a calcium fluoride $\left(\mathrm{CaF}_{2}\right)$ plate. As shown in Figure 2b, the ionization of iodine above $9.2 \mathrm{eV}$ served as a high pass filter (black line) and the transmittance of $\mathrm{CaF}_{2}$ as a low pass filter (blue line) making a bandpass at $9.7 \mathrm{eV}$ with the width of $0.7 \mathrm{eV}$ (blue region). When the $\mathrm{CaF}_{2}$ plate is replaced with strontium fluoride $\left(\mathrm{SrF}_{2}\right.$; red line), the resolution is improved to $0.4 \mathrm{eV}$ (red region). Since then, significant efforts have been devoted to improving the resolution 
(a)

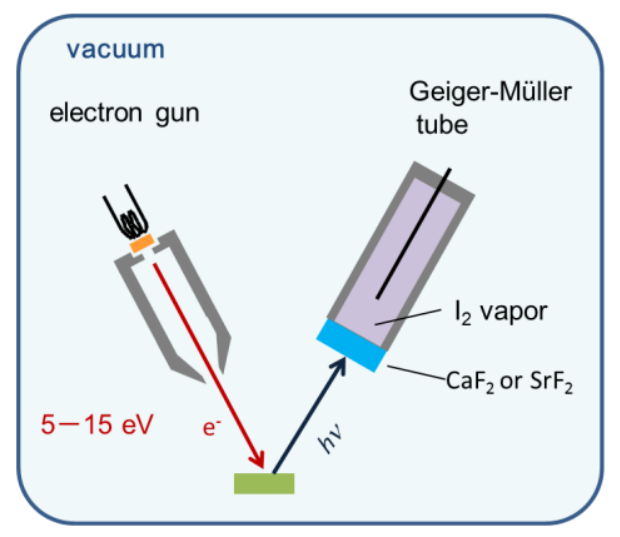

(c)

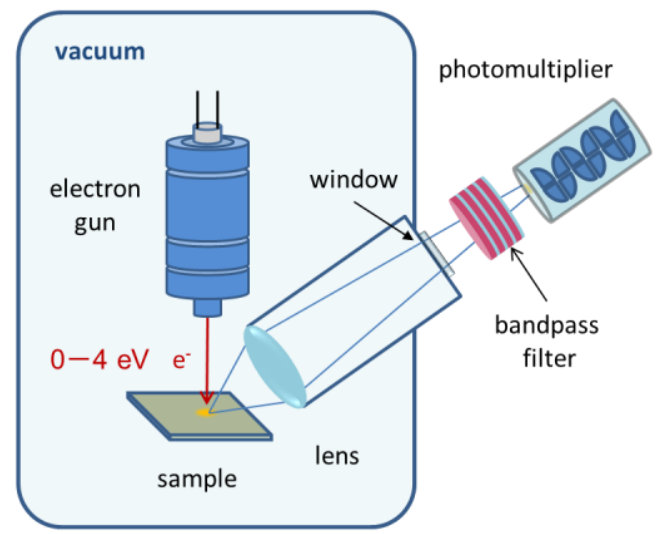

(b)
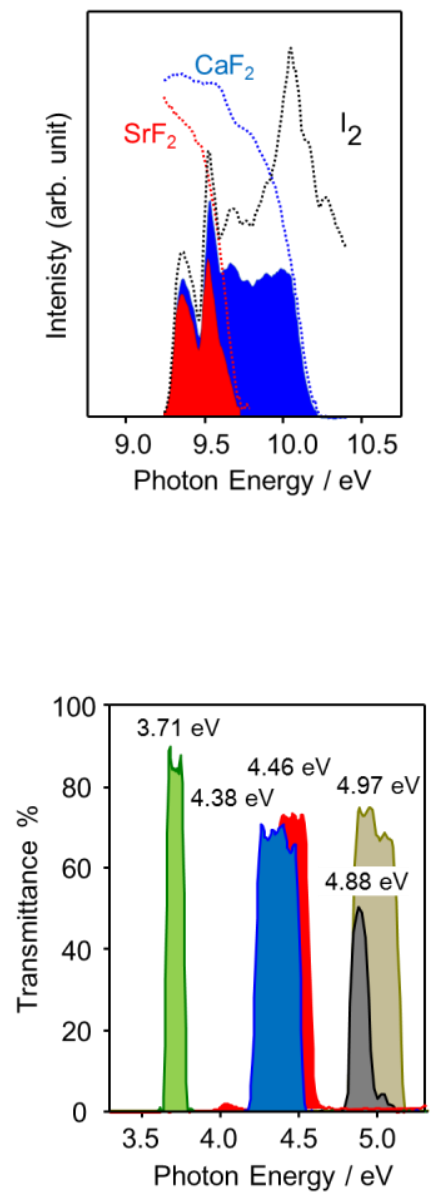

Fig 2:

Comparison of experimental apparatus and the sensitivity curves of the photon detector for IPES and LEIPS: (a) typical experimental setup for IPES and (b) sensitivity curve of the bandpass detector using iodine-filled Geiger-Müller tube [19, 20]; (c) schematic diagram of apparatus [28] and (d) transmittance of bandpass filters used for LEIPS where the center energies of bandpass are indicated.

by changing the filling gas, the filter material etc. So far the best resolution achieved is $0.084 \mathrm{eV}$ for the combination of acetone gas and $\mathrm{Sr}_{0.7} \mathrm{Ca}_{0.3} \mathrm{~F}_{2}$ plate [21].

The drawback of these bandpass detectors is that the resolution and center energy are inherently determined by the materials used. Further, an increase in resolution is accompanied by a loss in sensitivity limiting the resolution of practically useful bandpass detector to about $0.4 \mathrm{eV}$. Another drawback, which may be more serious, is damage in organic samples due to the electron bombardment. The center energies of these bandpass detectors are always around $10 \mathrm{eV}$, while the electron affinities of most organic semiconductors fall in the range between 0 and $5 \mathrm{eV}$ [22, 23]. Under these conditions the electron kinetic energy is scanned from 5 to $15 \mathrm{eV}$ for IPES measurements as shown in Figure 1. Electron irradiation of this energy range causes serious damage to organic samples [24]. Surprisingly, such IPES apparatus has been used for nearly four decades without fundamental improvement.

\section{Low energy inverse photoemission spectroscopy (LEIPS)}

Recently, low-energy inverse photoemission (LEIPS) has been reported [25] which simultaneously solves the two problems of conventional IPES. As shown in Figure 1, the electron energy is lowered below $4 \mathrm{eV}$, below the damage threshold of most organic molecules (about $5 \mathrm{eV}$ [26]). From energy conservation, the energy of emitted photons falls in the range between 2 and $5 \mathrm{eV}(600 \mathrm{~nm}$ and $250 \mathrm{~nm}$ in wavelength, i.e. the near ultraviolet (NUV) or visible range). The NUV or visible photons can be analyzed far more easily than VUV photons detected in the conventional IPES. The photons can be detected with high resolution and sensitivity using, for example, a grating spectrometer [27] or a multilayer interference bandpass filter [28]. A grating spectrometer allows more freedom in choosing photon energy and resolution, while the bandpass filter has an order of magnitude higher throughput. Since the low signal intensity is the main concern in IPES experiments, the bandpass filter is preferable in most cases. 
(a)

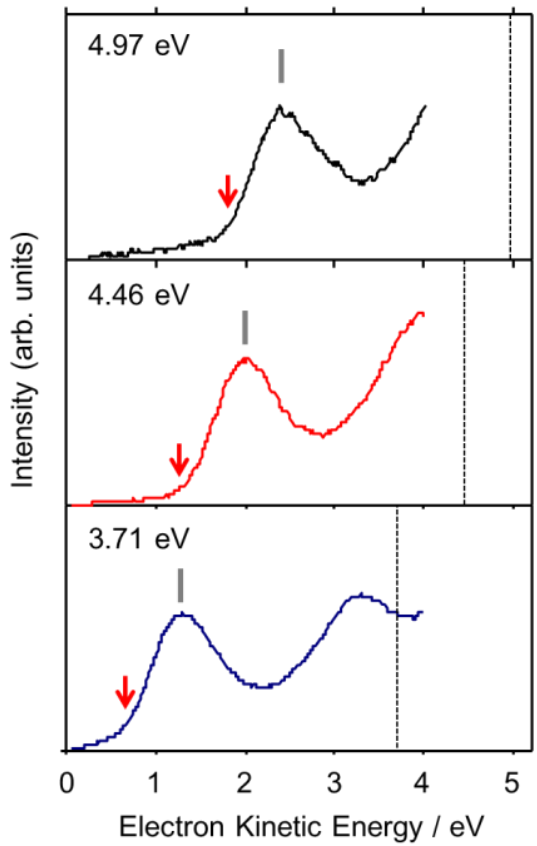

(b)
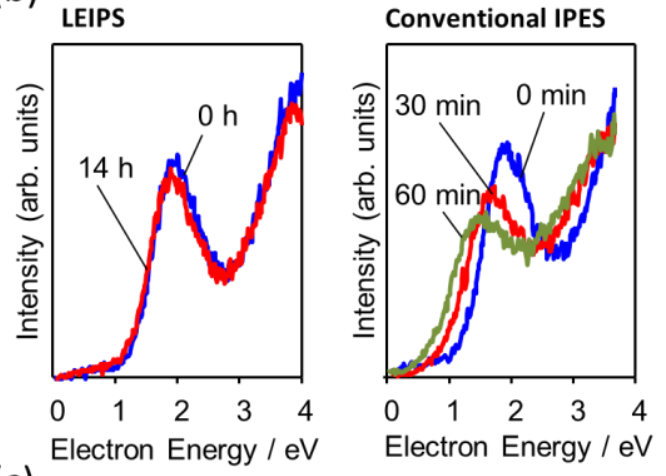

(c)

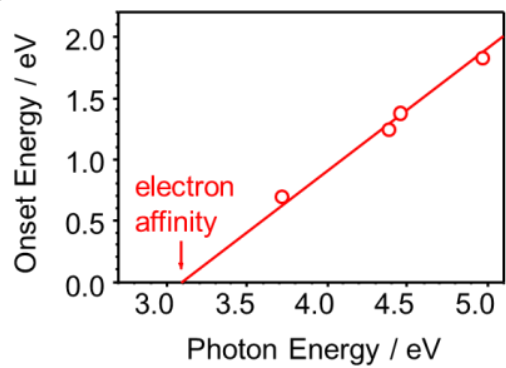

Fig 3:

LEIPS spectra and evaluation of electron affinity of copper phthalocyanine (CuPc [25]). (a) The spectra of $\mathrm{CuPc}$ taken at photon energies of $4.97 \mathrm{eV}, 4.46 \mathrm{eV}$ and $3.71 \mathrm{eV}$. (b) Time dependence of the spectra measured under typical condition of LEIPS (left panel) and similar condition to the conventional IPES (right panel) to access the sample damage. (c) The onset energies of the spectra plotted against the photon energy, fitted to a line with a slope of unity. The electron affinity of $\mathrm{CuPc}$ is determined from the intercept of the line.

A schematic diagram of the LEIPS apparatus is shown in Figure 2c [28]. Electrons from an electron source are focused onto the sample. The emitted NUV or visible photons are efficiently collected using a quartz lens. Since the NUV or visible photons are not absorbed by oxygen, photon detectors are placed in air; this greatly facilitates the construction and maintenance of the apparatus. The photons are analyzed using a multilayer interference bandpass filter and photomultiplier. The sensitivity curve of the bandpass filters are shown in Figure 2d. The center energy can be chosen from near ultraviolet to near infrared range and the resolution is between 0.1 and $0.25 \mathrm{eV}$. The resolution of the bandpass filter for LEIPS is 2-7 times better than the VUV bandpass detector shown in Figure 2b. The transmittance of photons is between $50 \%$ and $80 \%$ resulting in highly sensitive photon detection.

The overall resolution of LEIPS is determined by the resolution of photon detector and the energy spread of electrons. It is estimated to be $0.27 \mathrm{eV}$ from the spectrum of the Fermi edge of an Ag film when the resolution of the bandpass filter is $0.15 \mathrm{eV}$ [28]. This value is about a factor of two better than the previous IPES apparatus used for organic materials (about 0.5 $\mathrm{eV})$.

The LEIPS spectra of a typical organic semiconductor, copper phthalocyanine (CuPc), are shown in Figure 3a (the molecular structure of $\mathrm{CuPc}$ is shown in Figure 4).
The spectra are taken at several photon energies, 3.71 $\mathrm{eV}$ (with the resolution $0.09 \mathrm{eV}), 4.46 \mathrm{eV}(0.17 \mathrm{eV})$, and $4.97 \mathrm{eV}(0.23 \mathrm{eV})$. The spectrum shifts according to the photon energy meaning that the spectrum certainly reflects the density of unoccupied states and that the initial state effect is negligible. The spectra are also consistent with earlier IPES results [13, 29].

The observed first peak, derived from the lowest unoccupied molecular orbital (LUMO) of $\mathrm{CuPc}$, is about $1 \mathrm{eV}$ wide. The observed width is the convolution of the true spectrum and the instrumental function (or the overall resolution of the instrument). When the overall resolution is $0.3 \mathrm{eV}$, the true width of $1 \mathrm{eV}$ is only broadened by the measurement to $1.04 \mathrm{eV}$. This clearly indicates that the overall resolution is high enough to determine the electron affinity within an accuracy of $0.1 \mathrm{eV}$.

The radiation damage to $\mathrm{CuPc}$ is assessed by making prolonged measurements. In Figure 3b, a 1-hour scan is repeated and the spectrum stays unchanged even after 14 hours, showing that sample damage is negligible in LEIPS. In contrast, the spectral line shape is completely different after 30 minutes under the electron irradiation condition similar to conventional IPES. It is known that phothalocyanines are among the most durable molecules. Other molecules like polyacene are one order of magnitude, and the molecules with alkyl-chain is additional two orders of magnitude more sensitive to electron 
bombardment [30]. This means that most organic semiconductors will be damaged during conventional IPES measurements.

Since the resolution is improved and sample damage is negligible, the electron affinity is able to be determined precisely from the series of spectra in Figure 3a. The electron affinity is determined as the onset energy (indicated by the arrows in Figure 3a) with respect to the vacuum level (the dotted vertical lines). In Figure 3c, the onset energies are plotted as a function of photon energy and the electron affinity is determined as the intercept of the linear relation with the slope of unity. By this procedure, systematic errors can be suppressed. The electron affinity of $\mathrm{CuPc}$ is determined to be $3.09 \pm 0.05 \mathrm{eV}$.

\section{Examples of low-energy inverse photoemission spectroscopy}

\section{Electron affinities of typical organic semiconductors}

Using LEIPS, the electron affinities of widely-studies organic semiconductors were determined: perylene tetracarboxylic dianhydride (PTCDA), $\mathrm{C}_{60}$ [32], [6,6]-phenyl- $\mathrm{C}_{61}$-butyric acid methyl ester (PCBM) [32], zinc-phthalocyanine ( $\mathrm{ZnPc}), \mathrm{CuPc}$ [25] and pentacene [33]. The films of PCBM were prepared by spin-casting and while other compounds were by vacuum deposition on indium tin-oxide (ITO). The measurements were carried out at least at three different photon energies. The electron affinities are shown in Figure 4 together with their molecular structures. In LEIPS, the electron affinity can be determined with uncertainties as low as $0.1 \mathrm{eV}$. This accuracy of the value is much higher than for conventional IPES where the uncertainties are assumed to be as much as $0.7 \mathrm{eV}$ [23]. Note that the ionization energy depends (normally c.a. $0.1 \mathrm{eV}$ ) on the film structures such as crsytallinity, polymorphs [34, 35] and orientation of molecules [36]. Similar dependences are also expected in the electron affinities though such research has just started.

\section{Energy gap of pentacene [33]}

Since the ionization energies of organic solids have been extensively studied, the energy gap can be determined when the electron affinities is determined precisely [40][40]. The energy gap has been experimentally examined by various optical techniques[40]. [41]By comparing these values, electronic process in organic solid can be discussed in detail. The experimentally determined energy gaps also provide stringent tests for theoretical calculations [41].

The energy gap of pentacene, its molecular structure is shown in Figure 4, has been reported to be $1.8 \mathrm{eV}$ by photoabsorption spectroscopy, $2.2 \mathrm{eV}$ by photoconductivity measurements [42, 43], and $2.8 \mathrm{eV}$ if the charge-transfer (CT) exciton is assumed to interpret the electric field modulated absorption

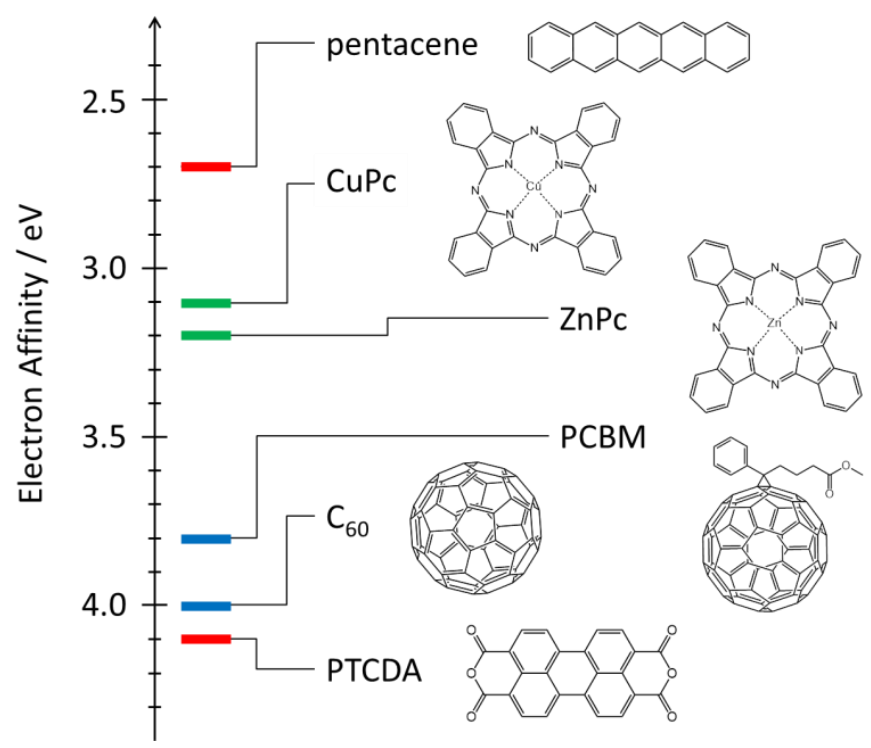

Fig 4:

Electron affinities and molecular structures of pentacene [33], copper phthalocyanine ( $\mathrm{CuPc}$ ) [25], zinc phthalocyanine $(\mathrm{ZnPc}),[6,6]$-phenyl- $\mathrm{C}_{61}$-butyric acid methyl ester (PCBM) [32], $\mathrm{C}_{60}$ [32] and perylene tetracalboxylic dianhydride (PTCDA) determined by LEIPS.

spectrum [44]. The LEIPS spectra of a 10-nm thick pentacene film were measured at the five different photon energies and the electron affinity was determined to be $2.70 \mathrm{eV}$ [33]. This gives the energy gap of $2.2 \mathrm{eV}$ using $4.9 \mathrm{eV}$ for the ionization energy. The value is in a good agreement with that obtained by photoconductivity measurement while larger by 0.4 $\mathrm{eV}$ than that of photoabsorption onset due to the exciton binding energy. The discrepancy with the CT exciton results suggests that the assignment of the $\mathrm{CT}$ exciton peak should be reconsidered.

Electron injection barrier between an electron conducting polymer and Au electrodes [37]

Energy barriers at the interface of an organic semiconductor device govern its performance. For the hole injection barriers, the interfacial electronic structure has been extensively studied [38, 39]. Similar study for the electrons can be done using LEIPS.

In an organic field effect transistor made using an electron conducting polymer, $\mathrm{P}(\mathrm{NDI} 2 \mathrm{OD}-\mathrm{T} 2)$, the activation energy was found to depend on the orientation of polymer and is higher in the film with the edge-on orientation with respect to the substrate surface. The reason is elucidated by measuring the electron affinities of polymers with face-on and edge-on orientations. Although the electron affinities of both face-on and edge on films are about $4.1 \mathrm{eV}$, the vacuum level are different leading to a difference in the electron injection barriers [38]. 


\section{Outlook}

Low-energy inverse photoemission spectroscopy (LEIPS) enables us to observe unoccupied states of solid materials with a resolution of $0.3 \mathrm{eV}$ and without radiation damage to the samples. The electron affinity of solid samples can be determined for the first time with the accuracy better than $0.1 \mathrm{eV}$ which meets the requirements in the research and development of organic electronics. A large advantage of organic electronics is that new materials can be tailored to the required physical and electronic properties by organic synthesis. This method will immediately be used in the development of new organic materials.

In the basic research, information about the valence states has been obtained using PES and the behavior of the hole in organic semiconductors has been clarified. Similar experiments for the unoccupied states and the electrons will be carried out using LEIPS. For example, the energy level alignment at the interface for unoccupied states and electron injection barrier will be examined as described in the previous section[38, 39]. The intermolecular band dispersion [45] is certainly the next target of LEIPS. The band dispersion of unoccupied states is closely related to electron transport [46, 47] though the conventional IPES cannot be applied because of sample damage and low resolution.

For such fundamental studies, even higher energy resolution is desirable. While the energy resolution is limited by the VUV bandpass detector and further improvement is difficult in the conventional IPES, the energy resolution of current LEIPS apparatus is limited by the energy spread of electrons. The electron energy spread will be narrowed soon to below $0.1 \mathrm{eV}$ by using an electron energy analyzer [48] resulting in an overall resolution of $0.1 \mathrm{eV}$. Such improvement of the experimental apparatus is expected to continue.

So far, LEIPS has been applied to organic materials in connection with the organic electronics. However, LEIPS is a more versatile technique in principle. It can be applied to adsorbates and surfaces of catalysts to elucidate catalytic behavior [46]. Since the method is non-destructive, biomolecules can be examined. Only the requirements for the sample materials are sufficient conductivity and vacuum compatibility.

\section{References}

1. Pauling, L., The nature of the chemical bond and the structure of molecules and crystals: an introduction to modern structural chemistry. 1960, New York: Cornell University Press.

2. Pope, M. and C.E. Swenberg, Electronic processes in organic crystals and polymers. 1999, New York: Oxford University Press.

3. Akamatu, H. and H. Inokuchi, On the electrical conductivity of violanthrone, iso-violanthrone, and pyranthrone. Journal of Chemical Physics, 1950. 18(6): p. 810-811.

4. Eley, D.D., Phthalocyanines as semiconductors. Nature, 1948. 162(4125): p. 819-819.

5. Vartanyan, A.T., Poluprovodnikovye svoistva organicheskikh krasitelei .1. ftalotsianiny. Zhurnal Fizicheskoi Khimii, 1948. 22(7): p. 769-782.

6. Tang, C.W. and S.A. Vanslyke, Organic electroluminescent diodes. Applied Physics Letters, 1987. 51(12): p. 913-915.

7. Tang, C.W., 2-Layer organic photovoltaic cell. Applied Physics Letters, 1986. 48(2): p. 183-185.

8. $\quad$ Green, M.A., et al., Solar cell efficiency tables (version 39). Progress in Photovoltaics, 2012. 20(1): p. 12-20.

9. In organic semiconductors, the valence (unoccupied) states correspond to the valence (conduction) bands in inorganic semiconductors, respectively. Since the edges of valence (unoccupied) states originate from the highest occupied molecular orbital, HOMO (the lowest unoccupied molecular orbital, LUMO) of the constituent molecules, they are often referred to HOMO (LUMO) level.

10. Fuggle, J.C. and J.E. Inglesfield, Unoccupied electronic states - fundamentals for xanes, eels, ips and bis - introduction. Topics in Applied Physics, 1992. 69: p. 1-23.

11. Seki, K. and K. Kanai, Development of experimental methods for determining the electronic structure of organic materials. Molecular Crystals and Liquid Crystals, 2006. 455: p. 145-181.

12. Bredas, J.L., J. Cornil, and A.J. Heeger, The exciton binding energy in luminescent conjugated polymers. Advanced Materials, 1996. 8(5): p. 447-452.

13. Hill, I.G., et al., Charge-separation energy in films of pi-conjugated organic molecules. Chemical Physics Letters, 2000. 327(3-4): p. 181-188.

14. Stricktly speaking, the energy released depends on the time scale. In the time scale of IPES process, only the electronic process is usually considered.

15. Johnson, P.D. and S.L. Hulbert, Inverse photoemission. Review of Scientific Instruments, 1990. 61(9): p. 2277-2288.

16. Hipps, K.W., Scanning tunneling spectroscopy (STS), in Handbook of Applied Solid State Spectroscopy, D.R. Vij, Editor. 2006, Springer. p. 305.

17. Soubatch, S., et al., Site-specific polarization screening in organic thin films. Physical Review Letters, 2009. 102(17).

18. Pendry, J.B., New probe for unoccupied bands at surfaces. Physical Review Letters, 1980. 45(16): p. 1356-1358.

19. Dose, V., VUV isochromat spectroscopy. Applied Physics, 1977. 14(1): p. 117-118.

20. Dose, V., Ultraviolet bremsstrahlung spectroscopy. Progress in Surface Science, 
1983. 13(3): p. 225-284.

21. Maniraj, M., et al., High energy resolution bandpass photon detector for inverse photoemission spectroscopy. Review of Scientific Instruments, 2011. 82(9): p. 093901.

22. Kahn, A., N. Koch, and W.Y. Gao, Electronic structure and electrical properties of interfaces between metals and pi-conjugated molecular films. Journal of Polymer Science Part B-Polymer Physics, 2003. 41(21): p. 2529-2548.

23. Djurovich, P.I., et al., Measurement of the lowest unoccupied molecular orbital energies of molecular organic semiconductors. Organic Electronics, 2009. 10(3): p. 515-520.

24. Tsutsumi, K., H. Yoshida, and N. Sato, Unoccupied electronic states in a hexatriacontane thin film studied by inverse photoemission spectroscopy. Chemical Physics Letters, 2002. 361(5-6): p. 367-373.

25. Yoshida, H., Near-ultraviolet inverse photoemission spectroscopy using ultra-low energy electrons. Chemical Physics Letters, 2012. 539-540: p. 180-185.

26. Boudaiffa, B., et al., Resonant formation of DNA strand breaks by low-energy (3 to $20 \mathrm{eV})$ electrons. Science, 2000. 287(5458): p. 1658-1660.

27. Yoshida, H., Low-energy inverse photoemission spectroscopy using a high-resolution grating spectrometer in the near ultraviolet range. Review of Scientific Instruments, 2013. 84(10): p. 103901.

28. Yoshida, H., Note: Low energy inverse photoemission spectroscopy apparatus Review of Scientific Instruments, 2014. 85: p. 016101.

29. Yoshida, H., K. Tsutsumi, and N. Sato, Unoccupied electronic states of 3d-transition metal phthalocyanines (MPc: $\mathrm{M}=\mathrm{Mn}, \mathrm{Fe}, \mathrm{Co}$, $\mathrm{Ni}, \mathrm{Cu}$ and $\mathrm{Zn}$ ) studied by inverse photoemission spectroscopy. Journal of Electron Spectroscopy and Related Phenomena, 2001. 121(1-3): p. 83-91.

30. Reimer, L., in Transmission electron microscopy: physics of image formation and microanalysis, Springer Series in Optical Sciences, P.W. Hawkes, Editor. 1997, Springer: Berlin.

31. Yoshida, H., Low-energy inverse photoemission spectroscopy using a grating spectrometer in the near ultraviolet range. Rev. Sci. Instrum., 2013. 84: p. 103901.

32. Yoshida, H., New experimental method to precisely examine the LUMO levels of organic semiconductors and application to the fullerene derivatives. MRS Symposium Proceedings, 2012. 1493: p. 295-301.

33. Han, W., et al., Electron affinity of pentacene thin film studied by radiation-damage free inverse photoemission spectroscopy. Applied Physics Letters, 2013. 103: p. 123303.

34. Sato, N., et al., Photoemission from an amorphous pentacene film. Chemical Physics,
1986. 109(1): p. 157-162.

35. Fukagawa, H., et al., Origin of the highest occupied band position in pentacene films from ultraviolet photoelectron spectroscopy: Hole stabilization versus band dispersion. Physical Review B, 2006. 73(24): p. 245310.

36. Duhm, S., et al., Orientation-dependent ionization energies and interface dipoles in ordered molecular assemblies. Nature Materials, 2008. 7(4): p. 326-332.

37. Fabiano, S., et al., Orientation-dependent electronic structures and charge transport mechanisms in ultrathin polymeric n-channel field-effect transistors. Acs Applied Materials \& Interfaces, 2013. 5(10): p. 4417-4422.

38. Ishii, H., et al., Energy level alignment and interfacial electronic structures at organic metal and organic organic interfaces. Advanced Materials, 1999. 11(8): p. 605-625.

39. Braun, S., W.R. Salaneck, and M. Fahlman, Energy-level alignment at organic/metal and organic/organic interfaces. Advanced Materials, 2009. 21(14-15): p. 1450-1472.

40. Krause, S., et al., Determination of transport levels of organic semiconductors by UPS and IPS. New Journal of Physics, 2008. 10: p. 085001.

41. Refaely-Abramson, S., R. Baer, and L. Kronik, Fundamental and excitation gaps in molecules of relevance for organic photovoltaics from an optimally tuned range-separated hybrid functional. Physical Review B, 2011. 84(7): p. 075144.

42. Silinshi, E.A., et al., Photoelectrical properties, energy level spectra, and photogeneration mechanisms of pentacene. Phys. Stat. Sol. (a), 1974. 25: p. 339.

43. Lang, D.V., et al., Amorphouslike density of gap states in single-crystal pentacene. Phys. Rev. Lett., 2004. 93(8): p. 086802.

44. Sebastian, L., G. Weiser, and H. Bassler, Charge transfer transitions in solid tetracene and pentacene studied by electroabsorption. Chem. Phys., 1981. 61: p. 125.

45. Ueno, N. and S. Kera, Electron spectroscopy of functional organic thin films: Deep insights into valence electronic structure in relation to charge transport property. Progress in Surface Science, 2008. 83(10-12): p. 490-557.

46. Hoffmann, R., Solids and surfaces: a chemist's view of bonding in extended structures. 1988, New York: VCH Publishers.

47. Ashcroft, N.W. and N.D. Mermin, Solid State Physics. 1976: Thomson Learning

48. Budke, M., et al., Inverse photoemission with energy resolution better than $200 \mathrm{meV}$. Review of Scientific Instruments, 2007. 78(8): p. 083903. 


\section{Online Abstract Figure}

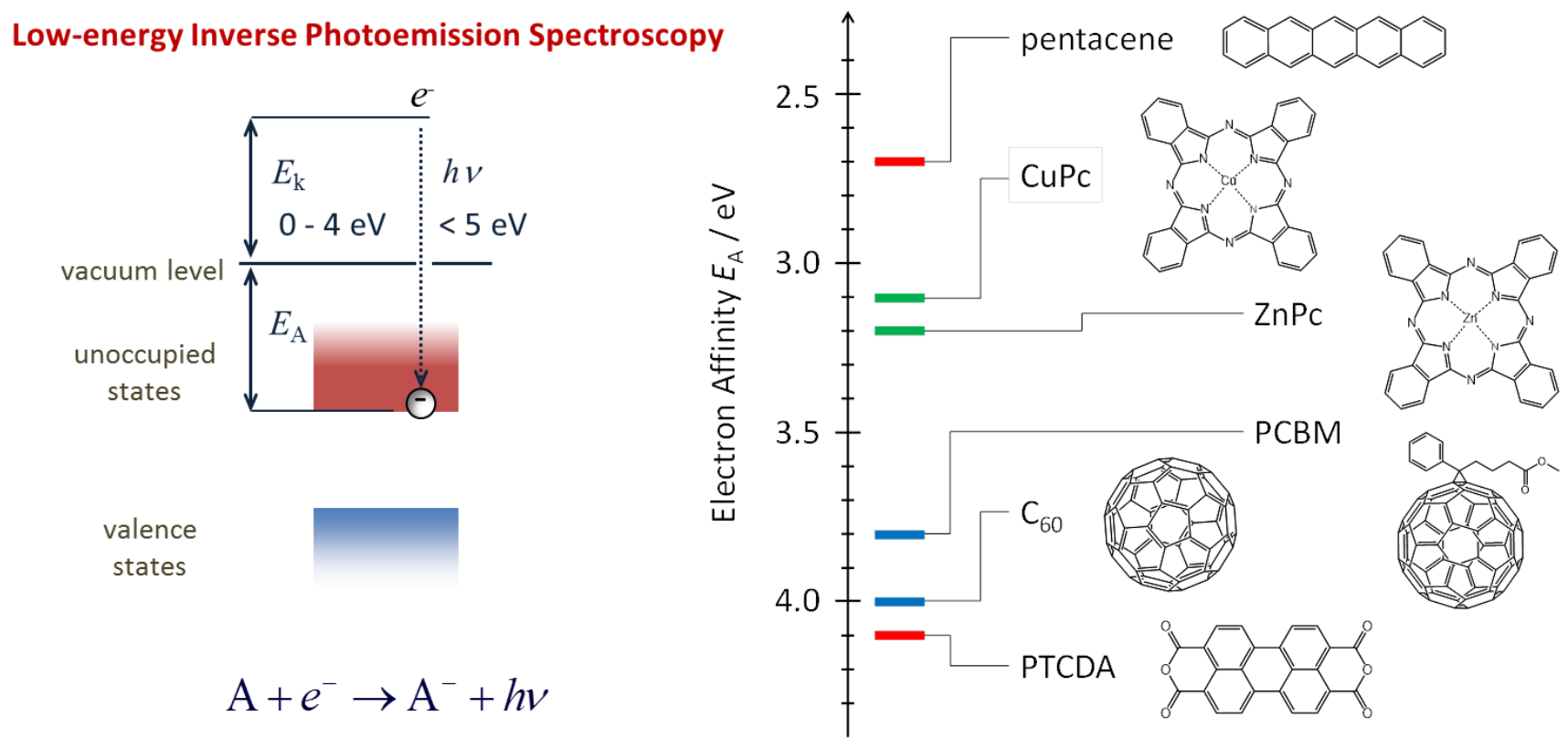

Yoshida H., "Measuring the electron affinity of organic solids: An indispensable new tool for organic electronics", Analytical and Bioanalytical Chemistry. 406(9-10), 2231-2237 (2014) http://dx.doi.org/10.1007/s00216-014-7659-1.

Present address (since April 1, 2015):

Hiroyuki Yoshida, Graduate School of Advance Integration Science, Chiba University, 1-33 Yayoi-cho, Inage-ku, Chiba 263-8522, Japan

Email: hyoshida@chiba-u.jp 